\title{
Ferromagnetic instability in PAAI in the sky
}

\author{
R. B. MacKenzie ${ }^{1}$, M. B. Paranjape ${ }^{1}$, and U. A. Yajnik ${ }^{2}$ \\ 1 Groupe de physique des particules, Département de physique, Université de \\ Montréal, Montréal, Québec, CANADA, \\ 2 Physics Department, Indian Institute of Technology Bombay, Mumbai, India \\ yajnik@iitb.ac.in
}

\begin{abstract}
We study an idealised plasma of fermions, coupled through an abelian gauge force $U(1)_{X}$, and which is asymmetric in that the masses of the oppositely charged species are greatly unequal. The system is dubbed PAAI, plasma asymétrique, abélien et idéalisé. It is argued that due to the ferromagnetic instability that arises, the ground state gives rise to a complex of domain walls. This complex being held together by stresses much stronger than cosmic gravity, does not evolve with the scale factor and along with the heavier oppositely charged partners simulates the required features of Dark Energy with mass scale for the lighter fermions in the micro-eV to nano-eV range. Further, residual $X$-magnetic fields through mixture with standard magnetic fields, can provide the seed for cosmic-scale magnetic fields. Thus the scenario can explain several cosmological puzzles including Dark Energy.
\end{abstract}

\section{Introduction}

There are several important unresolved issues in our current understanding of cosmology. Paramount among these are the problems of Dark Matter (DM) and Dark Energy (DE). Within the $\Lambda$-CDM model DM assists in galaxy formation and should be a gas of non-relativistic particles, while the issue of DE is closely tied to that of the cosmological constant [1], since data [2] suggest that its energy density is constant over the epochs scanned by the cosmic microwave background $(\mathrm{CMB})$. If treated as a dynamical phenomenon, DE demands an explanation for the equation of state $p=-\rho$ in terms of relativistic phenomena. From the point of view of naturalness, explaining a value of a dynamically generated quantity which is many orders of magnitude away from any of the scales of elementary particle physics or gravity is a major challenge. There are explanations that obtain such a sector as directly related to and derived from more powerful principles applicable at high scales [3] [4] [5]. On the other hand, extended and space filling objects, specifically domain walls as possible solutions to understanding Dark Energy have been proposed earlier in a variety of scenarios [6, 7][8][9] [10]. In this paper we pursue the latter approach, of invoking new species of particles and their interactions at the new low mass scale, agnostic of their connection to the known physics other than gravity. A more extensive discussion of the results reported here can be found in [11]. 
We consider a new sector of particles with interaction mediated by an unbroken abelian gauge symmetry denoted $U(1)_{X}$. The core of our mechanism involves the existence of a fermionic species that enters into a ferromagnetic state. As we will show, it is required to have an extremely small mass and hence an extremely large magnetic moment; we dub this species the magnino ${ }^{3}$, denoted $M$. We assume that the medium remains neutral under the $X$-charge due to the presence of a significantly heavier species $Y$ of opposite charge which does not enter the collective ferromagnetic state. The wall complex resulting from the formation of magnetic domains then remains mutually bound, and due to interaction strength much larger than cosmic gravity, remains frozen. The binding of the heavier species to this complex due to the requirement of $X$-electrical neutrality then ensures that these particles remain unevolving, and after averaging over the large scales of the cosmic horizon act like a homogeneous space filling medium of constant density.

It is possible to explain DM within the same sector, including possible dark atoms formed by such species [13][14] [15, 16]. This would also solve the concordance problem, that is, the comparable energy densities carried in the cosmological energy budget by the otherwise-unrelated components, DM and DE. Further, the $X$-electromagnetism is expected to mix kinetically with the standard electromagnetism. The existence of cosmic magnetic fields at galactic and intergalactic scales [17][18][19] is an outstanding puzzle of cosmology. Our mechanism relying as it does on spontaneous formation of domains of $X$-ferromagnetism has the potential to provide the seeds needed to generate the observed fields through such mixing.

In the following, in section 2 we motivate the origin of negative pressure for extended objects in cosmology. In 3 we discuss the calculation of the exchange energy for a spin polarised PAAI. Thus we motivate the possibility of occurrence of an extended structure of domain walls, and their metastable yet long lived nature. In section 4 we discuss the main results of our proposal, obtaining suggestive values for the masses and abundances for the scenario to successfully explain DE, and for the DM discussion we refer the reader to our longer paper [11]. In section 5 we obtain a restriction on the length scale of the domains for successful explanation of origin of cosmic magnetic fields from mixing with standard electromagnetism. After the conclusion in sec. 6 we also include a few salient questions from the audience and their answers in sec. 7 .

\section{Cosmic relics and the origin of negative pressure}

A homogeneous, isotropic universe is described by the Friedmann equation for the scale factor $a(t)$ supplemented by an equation of state relation $p=w \rho$. Extended relativistic objects in gauge theories in the cosmological setting[20] are known to lead to negative values for $w$ [21, 22]. A heuristic argument runs as follows. In the case of a frozen-out vortex line network, the average separation

\footnotetext{
3 The term magnino was earlier introduced in a different connotation in [33][34]
} 
between string segments scales as $1 / a^{3}$ but there is also an increment in the energy proportional to $a$ due to an average length of vortex network proportional to $a$ entering the physical volume. As such, the energy density of the network has to be taken to scale as $1 / a^{2}$, and we get the effective value $w=-1 / 3$. Likewise, for a domain wall complex, the effective energy density scales as $1 / a$ and $w=-2 / 3$. By extension, for a relativistic substance filling up space homogeneously, the energy density is independent of the scale factor, and has $w=-1$. In quantum theory this arises naturally as the vacuum expectation value of a relativistic scalar field. In the following, we consider a scenario that gives rise to a complex of domain walls whose separation scale is extremely small compared to the causal horizon and which remains fixed during expansion, and hence simulates an equation of state $p=-\rho$.

\section{$3 \quad$ Ferromagnetic instability of PAAI}

A system of fermions can be treated as a gas of weakly interacting quasi-particles in the presence of oppositely charged much heavier ions or protons which are mostly spectators and serve to keep the medium neutral. The total energy of such a system can be treated as a functional of electron number density, according to the Hohenberg-Kohn theorem. In a relativistic setting, it becomes a functional of the covariant 4-current, and hence also of the electron spin density [23]. In the Landau fermi liquid formalism the quasi-particle energy receives a correction from an interaction strength $f$ with other quasi-particles which can be determined from the forward scattering amplitude $\mathcal{M}$ [24]

$$
f\left(\mathbf{p s}, \mathbf{p}^{\prime} \mathbf{s}^{\prime}\right)=\frac{m}{\varepsilon^{0}(\mathbf{p})} \frac{m}{\varepsilon^{0}\left(\mathbf{p}^{\prime}\right)} \mathcal{M}\left(\mathbf{p} s, \mathbf{p}^{\prime} s^{\prime}\right),
$$

where $\varepsilon^{0}$ is the free particle energy and $\mathcal{M}$ is the Lorentz-covariant $2 \rightarrow 2$ scattering amplitude in a specific limit not discussed here. The exchange energy can equivalently be seen to arise as a two-loop correction to the self-energy of the fermion [25]. Using this $f$ one can compute the exchange energy $E_{\mathrm{xc}}$, as

$$
E_{\mathrm{xc}}=\sum_{ \pm \mathbf{s}} \sum_{ \pm \mathbf{s}^{\prime}} \int \frac{d^{3} p}{(2 \pi)^{3}} \frac{d^{3} p^{\prime}}{(2 \pi)^{3}} f\left(\mathbf{p s}, \mathbf{p}^{\prime} \mathbf{s}^{\prime}\right) n(\mathbf{p}, \mathbf{s}) n\left(\mathbf{p}^{\prime}, \mathbf{s}^{\prime}\right)
$$

and the effective quasi-particle energy is the kinetic energy of the quasi-particles with renormalised mass parameter $E_{\text {kin }}$ plus the spin-dependent exchange energy in a spin-polarised background. For this purpose it is necessary to calculate the self energy with a Feynman propagator in the presence of non-zero number density, and spin imbalance [26].

To set up a spin-asymmetric state, we introduce a parameter $\zeta$ such that the net density $n$ splits up into densities of spin up and down fermions as

$$
n_{\uparrow}=n(1+\zeta) \quad \text { and } \quad n_{\downarrow}=n(1-\zeta)
$$


Correspondingly, we have Fermi momenta $p_{F \uparrow}=p_{F}(1+\zeta)^{1 / 3}$ and $p_{F \downarrow}=p_{F}(1-$ $\zeta)^{1 / 3}$, with $p_{F}^{3}=3 \pi^{2} n$. The exchange energy was calculated in [26] and the final expression is too long to be quoted in this presentation. However the leading order expansions in $\beta=p_{F} / m$ for the fully polarised case $\zeta=1$ is[11]

$$
\begin{aligned}
& E_{\mathrm{kin}}(\zeta=1)=m^{4}\left\{\frac{\tilde{\beta}^{5}}{20 \pi^{2}}-\frac{\tilde{\beta}^{7}}{112 \pi^{2}}+O\left(\beta^{9}\right)\right\} \\
& E_{\mathrm{xc}}(\zeta=1)=-\alpha_{X} m^{4}\left\{\frac{\tilde{\beta}^{4}}{2 \pi^{2}}-\frac{7 \tilde{\beta}^{6}}{27 \pi^{2}}+O\left(\tilde{\beta}^{8}\right)\right\}
\end{aligned}
$$

where $\tilde{\beta}=2^{1 / 3} \beta$. The $\zeta=0$ case has same leading power laws with different coefficients. Thus the exchange energy tends to lower the quasi-particle energy parametrically determined by $\alpha$, with either $\zeta=0$ or $\zeta=1$ becoming the absolute minimum depending on $\beta$. For comparison, in this notation, the rest mass energy of the degenerate gas is $E_{\text {rest }}=m^{4} \beta^{3} /\left(3 \pi^{2}\right)$.

Exploring the energy expression presents three possibilities; $\zeta=1$ is not a minimum at all, $\zeta=1$ is a local minimum but $E(0)<E(1)$ i.e. a metastable vacuum and finally, $\zeta=1$ is the absolute minimum with $\zeta=0$ unstable vacuum. In Fig. 1 we have plotted the approximate regions of the three phases in the parameter space.

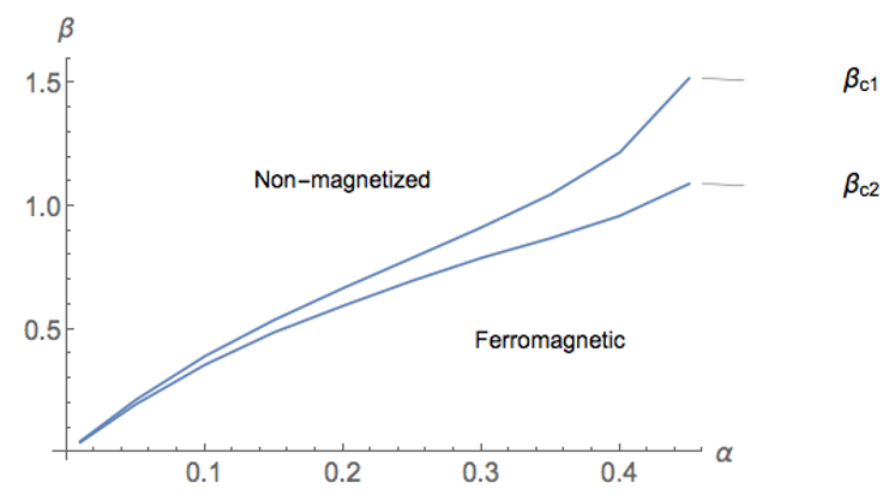

Fig. 1: Phase plot in the fine structure constant $\alpha$ vs $\beta=p_{F} / m$ plane showing the allowed region of spontaneous ferromagnetism

\subsection{Evolution and stability of domain walls}

We expect domain walls to occur in this spin polarised medium just like in ferromagnets. However due to the $S U(2)$ of spin being simply connected, the defects are not topologically stable and can unwind. However these processes are suppressed by a competition between the gradient energy and the extra 
energy stored in the domain walls, and there is a Ginzburg temperature $T_{G}$ [20] below which thermal fluctuations cannot destablise the walls trivially. The mechanism for destabilisation is then the one studied in detail in [27]. The rate for such decay is governed by an exponential factor $\exp (-B / \lambda)[28]$ where the exponent is the Euclidean action of a suitable "bounce" solution connecting the false and the true vacua [29]. On phenomenological grounds we need this complex to be stable for $\approx 10^{17} \mathrm{sec}$. The bounce $B$ is typically $\propto 1 / \lambda$ where $\lambda$ is a generic dimensionless coupling constant. Then large suppression factors $\sim 10^{-30}$ are natural for $\lambda \sim 0.01$. The other mechanism for disintegration of the DW network resides in the magnino gas becoming non-degenerate.

\section{A minimal model for Dark Energy}

We consider a hitherto unobserved sector with particle species we generically call $M$ and $Y$. They are assumed to be oppositely charged under a local abelian group $U(1)_{X}$ with fine structure constant $\alpha_{X}$. The mass $m_{M}$ of $M$ is assumed in the sub-eV range while the $Y$ mass $m_{Y}$ is assumed to be much larger. Charge neutrality requires that the number densities of the two species have to be equal, in turn this means that the Fermi energies are also the same. The hypothesis of larger mass is to ensures that $Y$ with Compton wavelength $M^{-1}<<p_{F}^{-1}$ does not enter into a collective magnetic phase.

We start our considerations at time $t_{1}$ when the temperature is just below $T_{G}$ so that the wall complex has materialised. The parameters of this wall complex are $\omega$, the thickness of individual walls and $L$, the average separation between walls. On the scale of the horizon, the wall complex behaves just like a space filling homogeneous substance. Further, due to the demand of neutrality, the heavier gas $Y$ cannot expand either, although it has no condensation effects. Let us denote the number density of the magninos trapped in the walls to be $n_{\text {walls }}^{X}$ and the remainder residing in the enclosed domains by $n_{\text {bulk }}^{X}$. Averaged (coarse grained) over a volume much larger than the $L^{3}$, this gives the average number density of the magninos to be

$$
\left\langle n^{X}\right\rangle=\frac{\omega}{L} n_{\mathrm{walls}}^{X}+\left(1-\frac{\omega}{L}\right) n_{\mathrm{bulk}}^{X}
$$

And from the neutrality condition we have

$$
\left\langle n^{X}\right\rangle=\left\langle n^{Y}\right\rangle
$$

Then we can demand that PAAI in this phase acts as the DE, so that assuming $Y$ to be non-relativistic, and ignoring other contributions,

$$
\rho^{Y} \approx m_{Y}\left\langle n^{Y}\right\rangle=\rho_{\mathrm{DE}}=2.81 \times 10^{-11}(\mathrm{eV})^{4}
$$

We can express the number density of $Y$ as a ratio of the number density $n_{\gamma}=3.12 \times 10^{-12}(\mathrm{eV})^{3}$ of photons, and set $\eta^{Y}=\left\langle n^{Y}\right\rangle / n_{\gamma}$. Then we can obtain conditions that determine the ratio

$$
\frac{m_{M}}{m_{Y}}=\frac{\beta^{Y}}{\beta} \approx\left(\eta^{Y}\right)^{4 / 3} \times 10^{-6} \ll 1
$$


These are the essential constraints determining the key parameters of our model. Then we find that $m_{M}$ ranges over $10^{-4}$ to $10^{-6} \mathrm{eV}$ corresponding to $\eta^{Y}$ ranging from $10^{-5}$ to $10^{-8}$; and $m_{Y}$ respectively ranges from $1 \mathrm{keV}$ to $1 \mathrm{GeV}$. Further, we can develop a corresponding multi-flavour dark sector so that Dark Matter can also be accommodated as neutral atoms of this sector. The details can be found in [11].

\section{Origin of cosmic magnetic fields}

The origin and evolution of galactic scale magnetic fields is an open question $[30,17]$. In particular the extent of seed magnetic field as against that generated by subsequent dynamics of the plasma is probably experimentally distinguishable [18][19]. In the present case, we can estimate the field strength of the $X$-magnetism in each domain, and is found to be

$$
B_{\mathrm{dom}} \approx\left(\frac{m_{M}}{\mathrm{eV}}\right)^{2}\left(\frac{\alpha_{M}}{\alpha}\right)^{1 / 2}\left(\frac{\beta}{0.1}\right)^{3} \times 2.2 \times 10^{-8} T
$$

Since the domain structure is completely random we expect zero large scale magnetic field on the average. Residual departure from this average can be estimated by assuming that the deviation from the mean grows as $\sqrt{N}$ as we include $N$ domains. Thus if the $X$-magnetic field in individual domains has the value $B_{\text {dom }}$ then on the scale of galactic clusters $L_{\text {gal }}$ it possesses a root mean square value $\overline{\Delta B} \equiv B_{\text {dom }}\left(L / L_{\text {gal }}\right)^{3 / 2}$.

Assuming $U(1)_{X}$ field mixes kinetically with standard electromagnetism through a term of the form $\xi F^{\mu \nu} F_{\mu \nu}^{X}$, the $\xi$ is well constrained from Supernova 1987A data to [31] $10^{-7}<\xi<10^{-9}$. The exact value of the seed required depends on the epoch being studied and other model dependent factors [32]. Let us consider the possibility of a seed of $10^{-30} \mathrm{~T}$ with a coherence length of $0.1 \mathrm{kpc} \sim 3 \times 10^{18}$ meter obtained with $\xi=10^{-8}$, i.e.,

$$
\overline{\Delta B}_{\text {seed }}=10^{-30} T \sim 10^{-8} \times\left(\frac{m_{M}}{\mathrm{eV}}\right)^{2}\left(\frac{\alpha_{M}}{\alpha}\right)^{1 / 2} \beta^{3}\left(\frac{L}{\text { meter }}\right)^{3 / 2} \times 10^{-40} T
$$

From this, representative values for $L$ for $\beta=0.1$ are in the range $10^{14}-10^{15}$ meter which is a fraction of the Milky Way size. A detailed treatment to estimate the residual fluxes on large coherence length scales could trace the statistics of flux values in near neighbour domains and the rate at which the magnetic flux could undergo percolation, providing perhaps a smaller value for $L$, comparable to the above estimate.

\section{Conclusions}

we have proposed the possibility of a negative pressure medium as arising from nothing more radical than a peculiar ground state of a pair of unequal mass 
fermion species interacting through an unbroken abelian gauge force. In an attempt to highlight the potential utility of the PAAI to cosmology, specifically to DE and to cosmic ferromagnetism, we have been agnostic about the earlier history of this sector. A study of temperature dependence of this phenomenon as also phenomenological inputs from the cosmic dawn data would help to sharpen this scenario.

\section{Questions from the audience}

Here we address two of the more important questions raised variously by several members of the audience, which we take the liberty to recapitulate and freely paraphrase. We gratefully acknowledge these inputs as having sharpened our understanding of our proposal.

Q1 The condensed state of the magninos seems to define a special frame of reference. Does that not conflict with the standard cosmology?

A1 The magnino and accompanying particles form a homogeneous relativistic gas at a high temperature just like the visible sector in the standard model of cosmology. They will be interacting with the standard sector at least gravitationally, and also possibly through other interactions during an early epoch so that the two define a common comoving frame. The new sector becomes "dark" only in the late universe. The emergent DW structure is then a comoving constant energy contribution.

Q2 What is the equation of the state of the spin polarised ground state? Intuitively any medium consisting of ordinary quasi-particles should be subject to evolution with the corresponding equation of state and will not simulate constant energy density.

A2 We have calculated the spin polarised medium if infinite, to satisfy $p=$ $-0.1 \rho$. However we note firstly that a negative value of effective $w$ implies a strongly coupled medium. Further, the domain wall structure would be immune to expansion since it exists by virtue of local stresses whose strength is many orders of magnitude greater than the local gravitational tidal force. For this reason we expect the DW complex to protect both itself and the strongly coupled quasi-particle gas from suffering tidal acceleration. Thus the energy density should remain constant, and averaged over an enormous number of domains, should be homogeneous .

\section{ACKNOWLEDGEMENTS}

We thank NSERC, Canada for financial support and the Ministère des relations internationales et la francophonie of the Government of Québec for financing within the cadre of the Québec-Maharashtra exchange. RBM and MBP also thank IIT Bombay for financial support and hospitality. 


\section{Bibliography}

[1] S. Weinberg, Rev. Mod. Phys. 61, 1 (1989).

[2] N. Aghanim et al. (Planck) (2018), 1807.06209.

[3] M. Li, X.-D. Li, S. Wang, and Y. Wang, Commun. Theor. Phys. 56, 525 (2011), 1103.5870.

[4] U. K. Dey, T. S. Ray, and U. Sarkar, Nucl. Phys. B928, 258 (2018), 1705. 08484.

[5] J. I. Kapusta, Phys. Rev. Lett. 93, 251801 (2004), hep-th/0407164.

[6] R. A. Battye, M. Bucher, and D. Spergel, Phys.Rev. D60, 043505 (1999), astro-ph/9908047.

[7] R. A. Battye and A. Moss, Phys. Rev. D76, 023005 (2007), astro-ph/ 0703744.

[8] L. Conversi, A. Melchiorri, L. Mersini-Houghton, and J. Silk, Astropart. Phys. 21, 443 (2004), astro-ph/0402529.

[9] A. Friedland, H. Murayama, and M. Perelstein, Phys. Rev. D67, 043519 (2003), astro-ph/0205520.

[10] U. A. Yajnik, EPJ Web Conf. 70, 00046 (2014).

[11] R. B. MacKenzie, M. B. Paranjape, and U. A. Yajnik (2019), 1901.00995.

[12] Note1, the term magnino was earlier introduced in a different connotation in [33][34]

[13] J. L. Feng, M. Kaplinghat, H. Tu, and H.-B. Yu, JCAP 0907, 004 (2009), 0905.3039.

[14] K. K. Boddy, M. Kaplinghat, A. Kwa, and A. H. G. Peter, Phys. Rev. D94, 123017 (2016), 1609.03592.

[15] J. M. Cline, Z. Liu, G. Moore, and W. Xue, Phys. Rev. D89, 043514 (2014), 1311.6468.

[16] J. M. Cline, Z. Liu, and W. Xue, Phys. Rev. D85, 101302 (2012), 1201.4858.

[17] R. M. Kulsrud and E. G. Zweibel, Rept. Prog. Phys. 71, 0046091 (2008), 0707.2783.

[18] R. Durrer and A. Neronov, Astron. Astrophys. Rev. 21, 62 (2013), 1303. 7121.

[19] K. Subramanian, Rept. Prog. Phys. 79, 076901 (2016), 1504.02311.

[20] T. W. B. Kibble, Phys. Rept. 67, 183 (1980).

[21] E. W. Kolb and M. S. Turner, The Early Universe (Addison-Wesley Pub. Co., 1990; revised 2003).

[22] S. Dodelson, Modern Cosmology (Addison-Wesley Pub. Co., 2003).

[23] A. K. Rajagopal and J. Callaway, Phys. Rev. B 7, 1912 (1973).

[24] G. Baym and S. A. Chin, Nucl. Phys. A262, 527 (1976).

[25] S. A. Chin, Annals Phys. 108, 301 (1977).

[26] B. X. Xu, A. K. Rajagopal, and M. V. Ramana, J. Phys. C: Solid State Physics 17, 1339 (1984).

[27] J. Preskill and A. Vilenkin, Phys. Rev. D47, 2324 (1993), hep-ph/9209210. 
[28] I. Yu. Kobzarev, L. B. Okun, and M. B. Voloshin, Sov. J. Nucl. Phys. 20, 644 (1975), [Yad. Fiz.20,1229(1974)].

[29] S. R. Coleman, Phys. Rev. D15, 2929 (1977), [Erratum: Phys. Rev.D16,1248(1977)].

[30] R. M. Kulsrud, Ann. Rev. Astron. Astrophys. 37, 37 (1999).

[31] S. Davidson, S. Hannestad, and G. Raffelt, JHEP 05, 003 (2000), hep-ph/ 0001179.

[32] L. M. Widrow, D. Ryu, D. R. G. Schleicher, K. Subramanian, C. G. Tsagas, and R. A. Treumann, Space Sci. Rev. 166, 37 (2012), 1109.4052.

[33] S. Raby and G. West, Phys. Lett. B200, 547 (1988).

[34] S. Raby and G. West, Phys. Lett. B194, 557 (1987). 